\title{
ARTICLE OPEN \\ Radiations effects in ISG glass: from structural changes to long-term aqueous behavior
}

\author{
Sylvain Peuget ${ }^{1}$, Magaly Tribet ${ }^{1}$, Sarah Mougnaud ${ }^{1}$, Sandrine Miro ${ }^{1}$ and Christophe Jégou ${ }^{1}$
}

The aim of the studies about the stability of nuclear glasses is to predict as accurately as possible their behavior over geological timescale. It requires the development of experimental methods to accelerate their irradiation and leaching ageing. This study focuses on the International Simple Glass behavior under irradiation by evaluating its structural and macroscopic property evolutions, and also its long-term chemical durability. Two irradiation ageing scenarios are considered: an alpha self-irradiation of a ${ }^{244} \mathrm{Cm}$-doped-ISG glass and an external irradiation with few MeV gold ions of non-radioactive ISG glass coupons. The results obtained from the Raman spectroscopy, density, and hardness measurements led to the conclusion that ballistic effects from the recoil nuclei are responsible of the changes with dose observed under alpha self-irradiation and that the ISG glass is a good surrogate of the French nuclear SON68 complex glass under such irradiation conditions. The assessment of the structural evolution with dose of this glass reinforces the mechanistic concepts about the glass response under alpha self-irradiation damage, where the glass response to a recoil nucleus event is the result of a very fast quenching of the damaged zone in the displacement cascade. Furthermore, the irradiation damage induced by gold ions does not fully mimic the ${ }^{244} \mathrm{Cm}$-doped glass evolution with nuclear dose, since the results of this simulation method overestimates the amplitude of the irradiation damage in the glass because the effects of the alpha particle are not included. Finally, this study demonstrates that the ISG glass water alteration at high-reaction progress is impacted by the glass damage, which indicates that the long-term glass chemical durability will depend on the glass structure when water arrives in contact.

npj Materials Degradation (2018)2:23; doi:10.1038/s41529-018-0044-3

\section{INTRODUCTION}

Electricity production by nuclear power plant is a carbon free energy source, but the development of a sustainable nuclear power requires the safe management of the nuclear wastes. Several countries have chosen the recycling of $U$ and $\mathrm{Pu}$ in MOX fuels associated to the vitrification of the ultimate wastes composed of fission products (FP) and minor actinides (MA). These glass canisters will be disposed in an underground geological repository. In most of the countries, the safety of such repositories relies on a multi-safety approach based on the properties of the host rock, of the metallic canisters and of the glass. Therefore the glass physical and chemical stabilities over geological timescale must be demonstrated which require the study of the glass response to the ionizing radiations emitted by the nuclear wastes together with its behavior under the chemical conditions encountered under geological disposal, i.e. mainly its chemical reactivity with respect to the water of the geological site. To that respect, numerous studies, performed on both aspects, were recently reviewed in ref. ${ }^{1}$ that highlighted the predominant role of alpha decays of minor actinides on the long-term radiation component and the need for improving the evaluation of the impact of such type of radiations on the glass leaching behavior.

The current study aims to assess the radiation response toward alpha decay irradiation of a simplified glass, named ISG (International Simple Glass) that was chosen by the international scientific community working on nuclear waste management, as a reference glass for the long-term aqueous leaching studies. ${ }^{2}$ This glass is also a simplified version, i.e. composed of six oxides, of the French R7T7 nuclear glass and was already partially studied with respect to radiation effects ${ }^{3-8}$ and leaching behavior. ${ }^{9-18}$ The characterization of the irradiated glassy state of ISG glass is of significant importance for the scientific community working in this international initiative about nuclear glass long-term behavior by offering a description of the glassy state after several thousand years of storage time, i.e. when water will access to the glass. Moreover, as ISG glass provides a simplified version of the French R7T7 glass, it allows the use of characterization tools to explore the glassy structure that were not easy to implement or interpret on the complex glass, such as NMR or Raman spectroscopy.

In the current article, we propose to simulate the radiation response of this glass to alpha decay accumulations by the two main experimental methods usually used. ${ }^{19,20}$ The first corresponds to the doping of the glass with a short half-life actinide $\left({ }^{244} \mathrm{Cm}\right)$ that induces in a few years a self-irradiation by alpha decays accumulation corresponding to the ageing of an industrial nuclear glass in several thousand years of storage time. The second method uses the external irradiation with heavy ions to simulate the nuclear collisions induced by the recoil nuclei of the alpha decays. Indeed it was suggested on complex SON68 glass (laboratory simulated version of R7T7 glass) that the evolution of the glass properties with alpha decay dose was controlled by the accumulation of the nuclear collisions arising from the recoil nuclei. $^{20}$ Therefore, this external irradiation method seems to correctly simulate the material transformation with dose.

${ }^{1}$ CEA, DEN, DE2D, SEVT, F-30207 Bagnols-sur-Cèze, France

Correspondence: Sylvain Peuget (sylvain.peuget@cea.fr)

Received: 16 March 2018 Revised: 4 June 2018 Accepted: 5 June 2018

Published online: 20 August 2018 

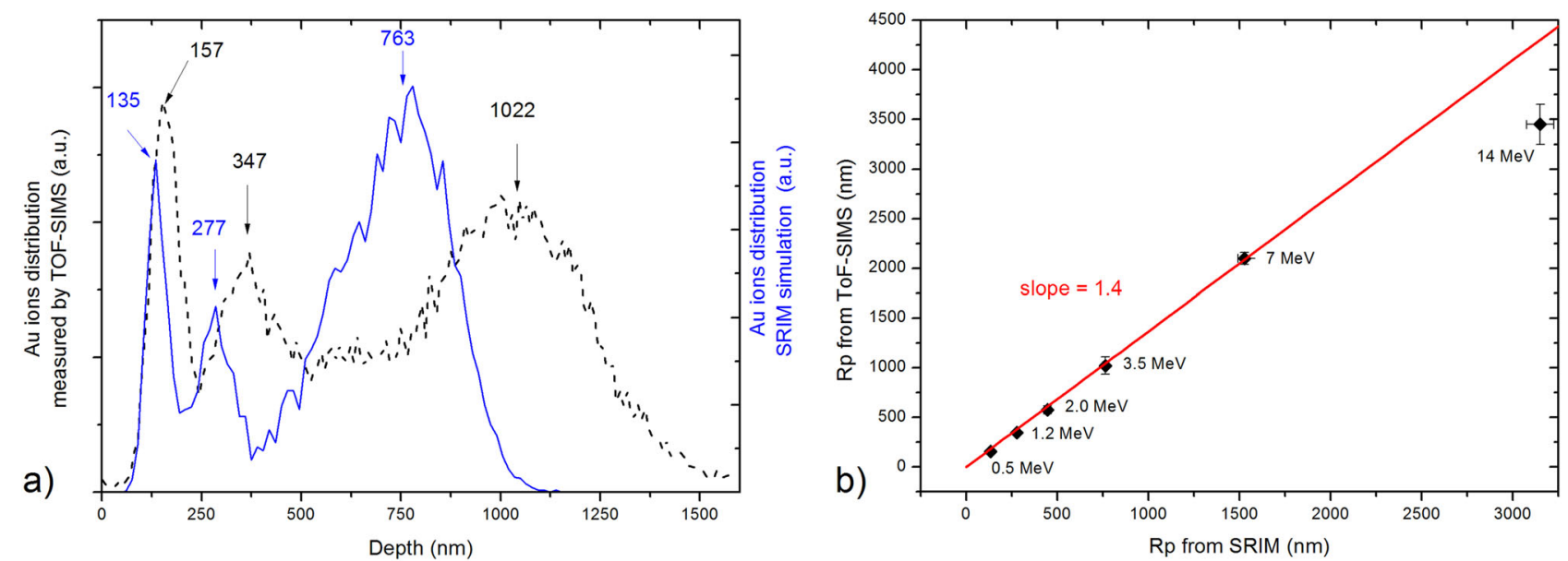

Fig. 1 (a) Au ions distribution from TOF-SIMS measurement (black) and SRIM simulation (blue) associated to the multi-energy Au ions irradiation (0.5-3.5 MeV), (b) Evolution of the experimental gold ions range, measured by ToF-SIMS, versus the expected gold ions range from SRIM simulation. The data from 0.5 to $3.5 \mathrm{MeV}$ were obtained on the same sample irradiated in this study in multi-energy mode at a fluence of $5.5 \times 10^{14}$ ions $/ \mathrm{cm}^{2}$. The data for 7 and $14 \mathrm{MeV}$ Au ions irradiations were measured on two ISG glasses irradiated in previous studies. ${ }^{45,46}$ The associated error bar for each Rp value is determined from the standard error associated to the gaussian fit of the gold distribution profiles

Nevertheless such conclusion relies on one study performed on the complex SON68 glass with only two macroscopic parameters affected by the irradiation that could be compared: the glass hardness and its density. The current study aims thus to confirm such a conclusion by focusing on the ISG glass and by evaluating its structural and macroscopic property behaviors under both types of irradiation conditions. Moreover, the impact of a prior irradiation on the further glass chemical durability is also studied.

\section{RESULTS}

Range of gold ions in ISG glass

The comparison of the damage induced by alpha decay selfirradiation and $\mathrm{Au}$ ions irradiation requires the calculation of the local damage associated to the external irradiation conditions. Therefore, the Au implantation profile was characterized by ToFSIMS in order to compare the Au ions implanted range to the one predicted by the SRIM simulation ${ }^{21}$ (Fig. 1) and correct the local damage evaluation from SRIM according to the measured implanted range. The results show that the SRIM 2008 simulation underestimates the penetration depth of $\mathrm{Au}$ ions into the glass. The difference of range is large and cannot be explained by the density change of the glass during the irradiation or by the uncertainty of the Au ions energy. This effect has already been described in the literature and is linked to an overestimation of the electronic stopping power of heavy ions in light matrices. ${ }^{22}$ Therefore, up to $7 \mathrm{MeV}$, a constant correction factor equal to 1.4 has thus been considered to calculate both the real energy deposition values from the SRIM simulation data, and the corresponding dose values (in Gy and dpa). Then, when the gold ion implantation energy increases (14 MeV gold ions), this underestimation tends to diminish as presented in ref. ${ }^{22}$.

\section{Structural evolution with irradiation}

Both types of irradiations, alpha decay self-irradiation (Fig. 2a) or Au ions irradiation (Fig. 2b), induce a change in the glass structure that has been characterized by the Raman spectroscopy, whose characteristics are qualitatively the same, except for the region above $1250 \mathrm{~cm}^{-1}$ where the Raman signal from the $\mathrm{Cm}$-ISG glass, i.e. the B-O stretching band, is covered by the curium luminescence signal. Indeed the first main vibration band at around $490 \mathrm{~cm}^{-1}$, called R band and assigned to the bending and rocking modes of $\mathrm{Si}-\mathrm{O}-\mathrm{Si}$ bonds, ${ }^{23}$ is shifted to higher wavenumbers with the irradiation dose. It corresponds to a decrease of the mean angle in between the silica tetrahedra. Moreover the contribution at around $600 \mathrm{~cm}^{-1}$, called $D_{2}$ band and assigned to the vibration of three membered $\mathrm{SiO}_{4}$ rings, ${ }^{24}$ also increases for both irradiation conditions together with an important change of the shape of the $Q_{n}$ band situated in between 850 and $1200 \mathrm{~cm}^{-1}$. This band is usually assigned to the stretching of the $\mathrm{Si}-\mathrm{O}$ bond which is affected by the surrounding of the $\mathrm{SiO}_{4}$ tetrahedra. In alkali silicates and borosilicates, it is usually considered that 5 contributions can be observed at around $850,930,990,1100$, and $1200 \mathrm{~cm}^{-1}$ corresponding to the vibration of $\mathrm{SiO}_{4}$ tetrahedra connected, respectively, to 4, 3, 2, 1, and 0 nonbridging oxygen (NBO) atoms. ${ }^{25,26}$ In ISG glass, two main contributions are easily seen at around 980 and $1080 \mathrm{~cm}^{-1}$, that we will call $\mathrm{Q}_{a}$ and $\mathrm{Q}_{b}$. As already discussed in ref. ${ }^{4}$ according the glass composition, to the position of the ${ }^{29} \mathrm{Si} \mathrm{NMR}$ spectra, and to the recent results of modeling of ISG glass structure, ${ }^{27}$ it is obvious that no $Q_{2}$ units exist in the glass and that the origin of the main contribution in the $Q_{n}$ band of ISG glass cannot be explained by a classical $Q_{2}$ unit involving two NBOs. We have already proposed that this contribution should be associated to the existence of some $\mathrm{Q}_{4}(2 \mathrm{~T})$ units, corresponding to a fully polymerized silica tetrahedral but bonded to two glass formers other than Si atoms, i.e. $\mathrm{B}, \mathrm{Al}$, or $\mathrm{Zr}$, that affect the $\mathrm{Si}-\mathrm{O}$ vibration in such tetrahedra similarly to the one in a pure $Q_{2}$ unit. Fig. $2 a, b$ shows that both irradiation conditions induce a decrease of the $Q_{a}$ contribution with dose, reflecting a change of the surrounding of some of the $\mathrm{SiO}_{4}$ tetrahedra. It is also noticeable that for both irradiation conditions, the Raman spectra change up to a saturation state, obtained after a certain dose level, reflecting the reach a new glassy state that is not affected anymore by the increase of the irradiation dose.

So as to quantitatively compare the structural changes induced by the two irradiation conditions, the decrease of $\mathrm{Q}_{a}$ band is plotted in Fig. 2c, d versus the deposited nuclear and electronic doses respectively, i.e. by the two mechanisms of radiation/matter interaction (nuclear and electronic collisions) that coexist for both irradiations. ${ }^{28}$ The figure illustrates that both $\mathrm{Q}_{a}$ decreases with dose follow a direct impact model (an exponential law, i.e. $\left.\Delta \mathrm{Q}_{a}=\mathrm{A}\left(1-e^{-\mathrm{B} \times \text { dose }}\right)\right), \mathrm{A}$ is the saturation change and $\mathrm{B}$ is the impact parameter), with the same variation with nuclear dose (same B parameter of around $5 \times 10^{-8} \mathrm{~Gy}^{-1}$ ), and a shift by more than one order of magnitude with electronic dose. It suggests that the nuclear collisions control the structural transformation of the 

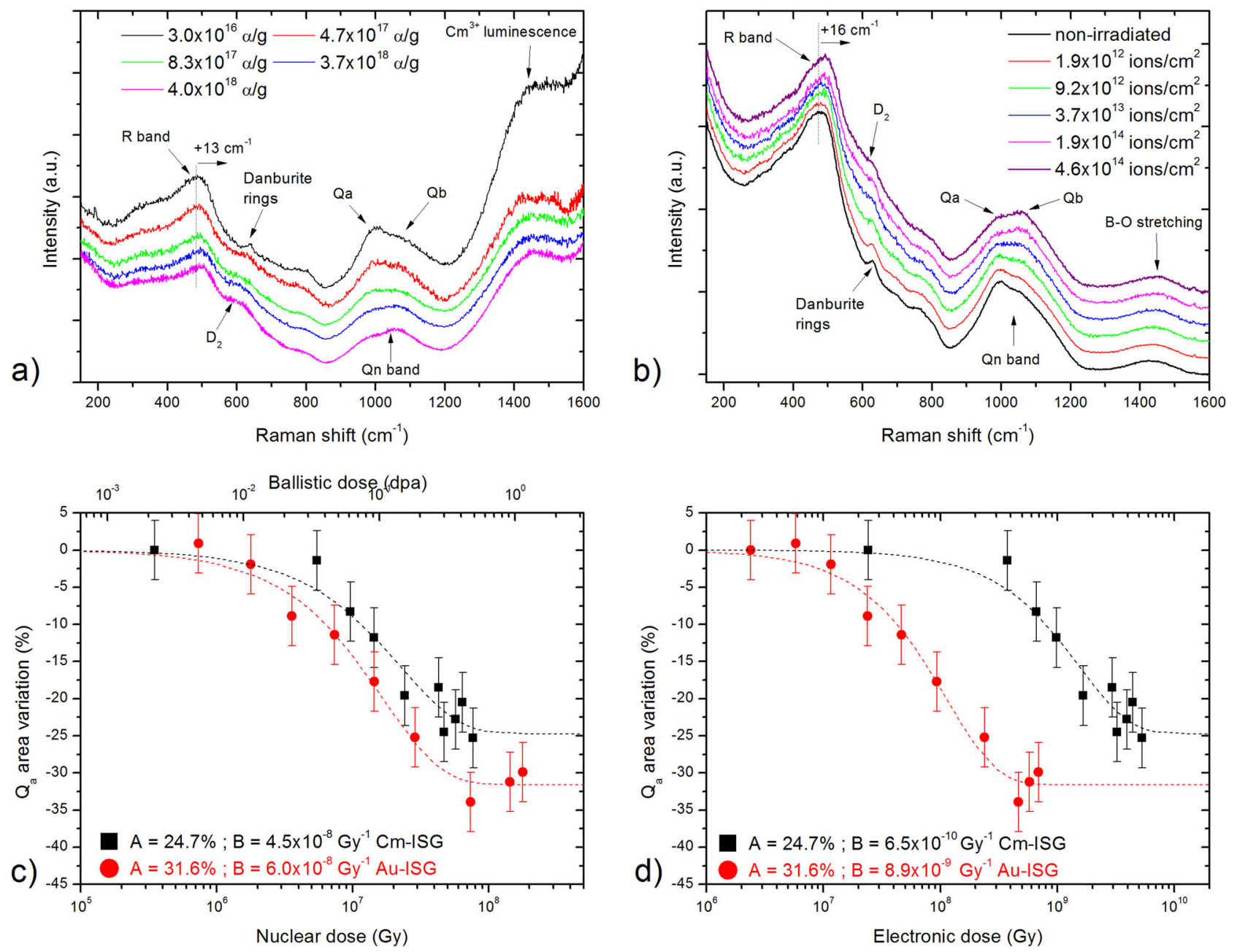

Fig. 2 (a) Raman spectra of Cm-ISG glass (5 alpha decay doses). (b) Raman spectra of Au-ISG glass (non-irradiated and 5 irradiation fluences). (c) $\mathrm{Q}_{a}$ area variation versus nuclear dose for Cm-ISG (black) and Au-ISG (red), (d) $\mathrm{Q}_{a}$ area variation versus electronic dose for Cm-ISG (black) and Au-ISG (red). The dotted lines are fits of experimental data with an exponential function $A(1-\exp (-B \times D o s e)$, in which $A$ is the value at saturation and $B$ is the impact parameter. The associated errors for the $Q_{a}$ values are related to the standard error of the Gaussian fit of the $Q_{a}$ contribution in the $\mathrm{Q}_{n}$ band of the Raman spectra

glass. It also shows that the $\mathrm{Q}_{a}$ decrease at saturation (A parameter) is lower for alpha decay self-irradiation $(-24.7 \%)$ than for Au irradiation (-31.6\%).

\section{Macroscopic property changes}

The effect of both irradiations on the intrinsic macroscopic properties of the ISG glass was characterized by density and hardness measurements. Their changes are shown in Fig. 3a, b, i.e. a decrease according to an exponential law with same variation with nuclear dose (B parameter) for both irradiation conditions. As for structural parameters, the changes do not match when plotted versus the electronic dose (not shown for the sake of brevity) which also suggests that nuclear collisions are responsible of the properties changes. It is interesting to notice that the changes of hardness are observed at a slightly lower dose level (higher $B$ values of around $1.5 \times 10^{-7} \mathrm{~Gy}^{-1}$ ) than for density and structural changes for which the changes with dose follow the same exponential variation (same $B$ parameter values of around $5 \times$ $10^{-8} \mathrm{~Gy}^{-1}$ ). It suggests that the density and structural changes are associated and that the hardness changes could be related to another mechanism of transformation. As for structural changes, alpha decay self-irradiation induces lower changes at saturation ( $A$ parameter) of density and hardness than $\mathrm{Au}$ ion irradiation.
These results are the first experimental data obtained on an alpha self-irradiated actinide doped ISG glass and cannot be compared with other data from the literature. Because ISG glass is a simplified version of the SON68 glass (same molar ratio of the constitutive oxides), we can try to compare these results with those obtained on the various actinide doped SON68 glasses studied in the literature. ${ }^{20,29-32}$ The density and hardness changes at saturation are quite similar for both glasses with a swelling of around $0.5 \%$ and a hardness decrease of around 30\% for SON68 glass. It shows that ISG glass seems to be a good simple glass to simulate the macroscopic behavior under alpha self-irradiation of complex nuclear SON68 glass.

The changes of macroscopic properties induced by the gold ions irradiation can be compared with the recent results obtained by Karakurt with $7 \mathrm{MeV} \mathrm{Au}$ ions irradiation ${ }^{8}$ which showed a decrease of hardness of similar amplitude, $-38 \%$ compared to $-36 \%$ in our study, and a decrease of density that seems to be higher, $-2.7 \%$ compared to $-1.6 \%$ in this study. Nevertheless, the density change measured by Karakurt should thus be corrected due to the lower ion range estimation by SRIM simulation. By taking the ion range correction factor of 1.4 (Fig. 1), the density change from Karakurt study is thus of around $1.9 \%$ which is closer to the value we obtained. Therefore, it shows the importance of the range measurement of the implanted ions to evaluate the 

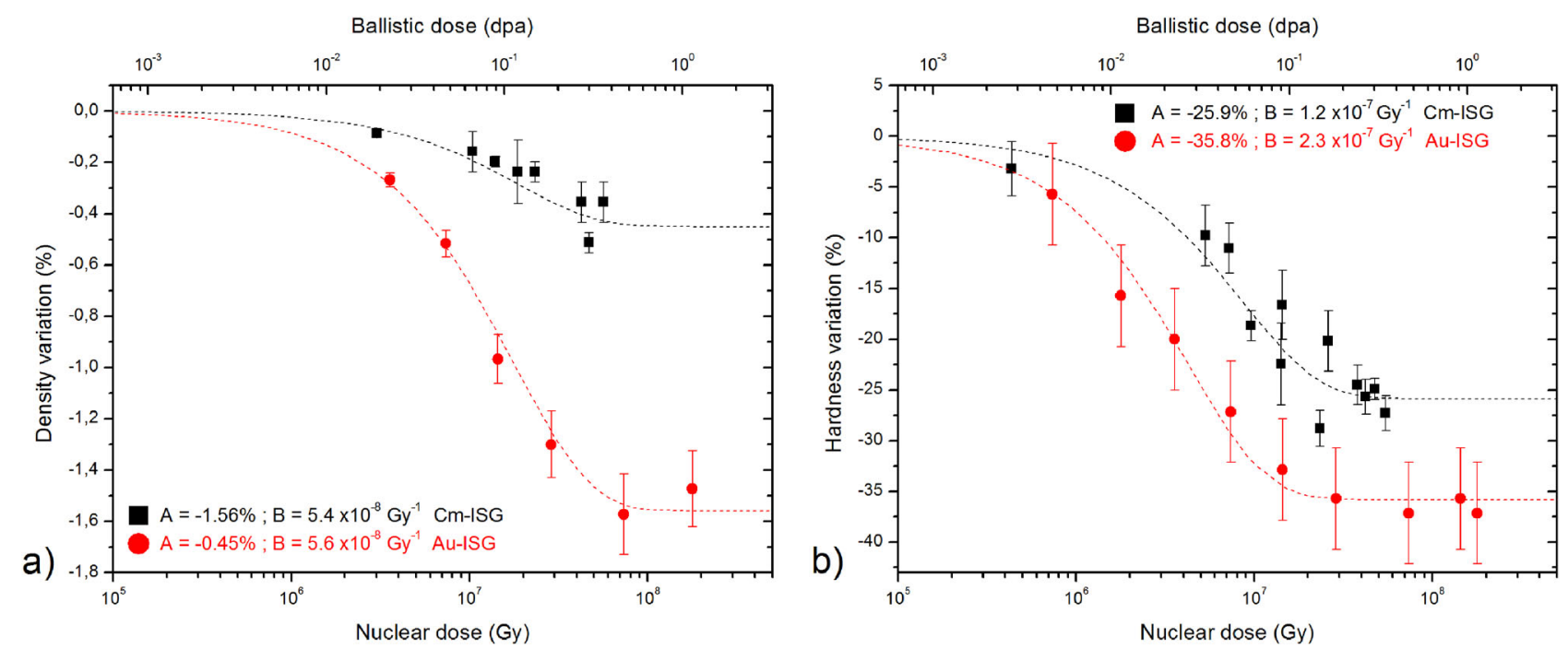

Fig. 3 Density (a) and hardness (b) variations versus nuclear dose for Cm-ISG glass (black squares) and Au-ISG (red circles). The plotted data are the mean values obtained experimentally and the associated errors bars represent the corresponding standard deviation. The dotted lines are fits of experimental data with an exponential function $A(1-\exp (-B \times D o s e)$, in which $A$ is the value at saturation and $B$ is the impact parameter
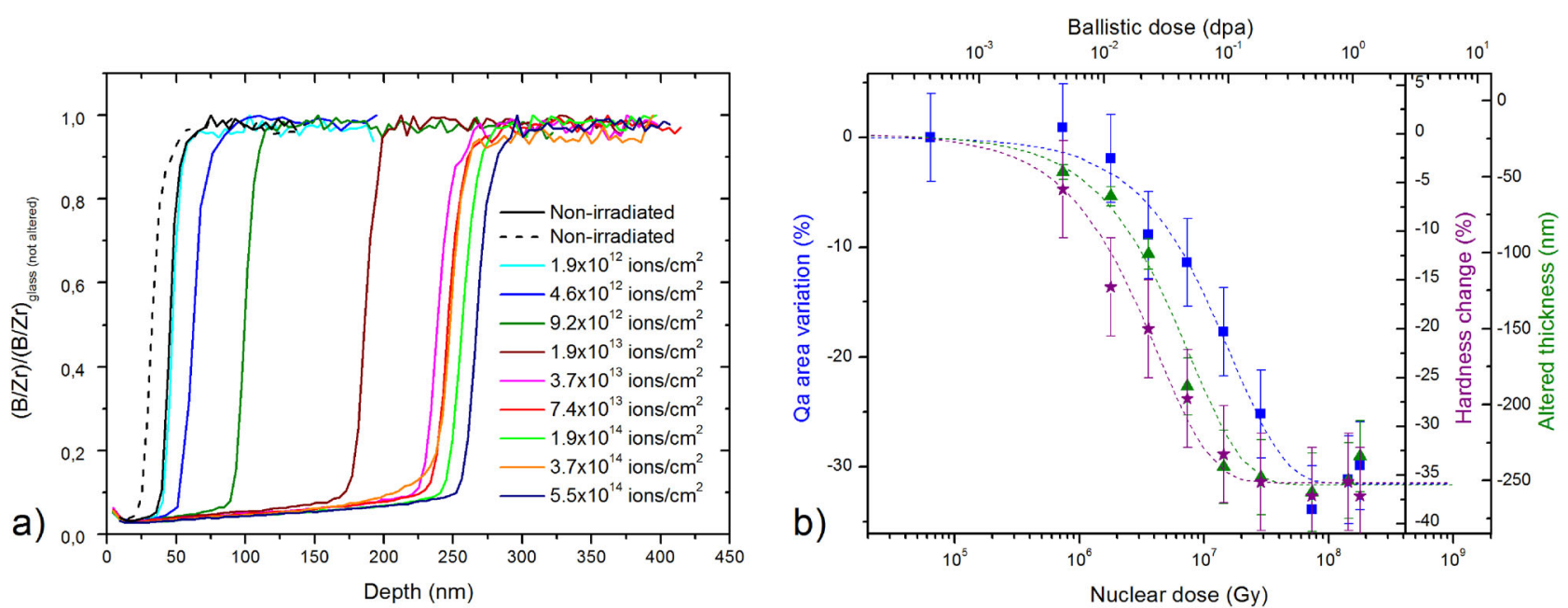

Fig. 4 (a) Normalized Boron concentration profiles (methods from ref. ${ }^{10}$ ) in Au-ISG glass samples, irradiated at different fluences and analyzed in irradiated zones (colored lines) or non-irradiated zones (black lines), altered for 13 days. (b) $\mathrm{Q}_{a}$ area variation (blue squares), hardness change (purple stars) and altered thickness (green triangles) versus nuclear dose for Au-ISG glasses. The associated errors bars represent the standard deviation of the experimental data (altered thickness and hardness) or the standard error associated to the Gaussian fit (Qa). The dotted lines are fits of experimental data with an exponential function $A(1-\exp (-B \times$ Dose), in which $A$ is the value at saturation and $B$ is the impact parameter

density changes under external irradiation, and the need to measure the electronic energy loss of heavy ions in a light matrix so as to correct the data-base used by SRIM. The accurate simulation of material transformation under external irradiation would be improved by such measurements.

The effect of these irradiations on the reactivity of the glass with respect to water was evaluated by leaching tests performed at high surface to volume ratio to simulate the long-term alteration conditions (i.e. high-reaction progress). The glass alteration leads to the formation of an alteration layer depleted in some elements, mainly boron and sodium which are considered as tracers. ${ }^{33}$ To probe the irradiation effects, we focused on the alteration layer thickness dependence with the Au ions irradiation fluence and alteration time, based on boron profiles. Figure 4a presents the normalized boron profiles as a function of depth, for various irradiated fluences and an alteration time of 13 days. The profiles in non-irradiated zones (black lines) and in the irradiated one for fluence 1 are quite similar within the experimental errors (around $10 \mathrm{~nm}$ ). In the irradiated zones for fluences $2-5$, the profiles are gradually shifted toward greater depth. For fluences 6-10, profiles are all situated in the same range of depth $(240 \pm 20 \mathrm{~nm})$, regardless of irradiation fluence. The abrupt transition in each profile corresponds to the interface between the alteration layer (depleted in boron) and the initial glass, and allows to calculate the alteration layer thickness. The variation of this alteration thickness with nuclear dose is presented in Fig. 4b (green triangles) and can also be correctly fitted with an exponential law. These results show that the long-term glass chemical durability is clearly sensitive to glass modifications due to a prior irradiation and follows the same trends as the glass properties and structural evolutions with ballistic irradiation dose. These results on the effect of the glass damage on the long-term glass 
alteration behavior are the first one described in the literature. Until now, alpha decay irradiation impact on glass leaching behavior focused on the initial alteration rate of SON68 complex glasses, measured by Soxhlet-mode dynamic leach tests at $373 \mathrm{~K}$ from both actinide-doped glasses and externally irradiated glasses with heavy ions. ${ }^{34,35}$ And, in every case (doped or externally irradiated complex glasses), no effect of irradiation on this initial alteration rate was evidenced. This suggests that the main mechanisms involved in both leaching steps are different and/or that the glass composition has an important role on the leaching glass response to irradiation.

Moreover it can be noticed (Fig. 4b) that the altered layer thickness variation with dose is "midway" between the hardness and structural changes. It is not surprising that the structural and volume changes (density changes) are related, both being proportional to the material damaged fraction. For the hardness change, it must be kept in mind that the hardness is related to the plastic flow of the glass at a macroscopic scale and that its changes could not be directly proportional to the material damaged fraction. Indeed, once the damaged fraction reaches the volume percolation threshold $(\sim 0.3)$, the plastic response of the glass should be significantly affected by the irradiation and consequently, its variation with dose should be observed before the one of volume changes (density) and structural parameters. For the alteration layer thickness variation with dose, a correlation with the structural parameters should exist. Nevertheless as it evolves in between the hardness and structural changes, it suggests that a percolation threshold could be involved in the leaching response of the irradiated glass or that other structural changes not identified yet, that saturates before the one studied here, could control the leaching response of the irradiated glass.

\section{DISCUSSION}

The final aim of the studies about the stability of nuclear glasses is to predict as best as possible their behavior over geological timescale. It requires the development of experimental simulation methods to accelerate their irradiation and leaching ageing. In this study, we have compared the behavior of ISG glass with respect to alpha decay self-irradiation and $\mathrm{Au}$ ions irradiation to simulate the effect of the recoil nuclei of alpha decays. The first method is considered to be the best representation of the ageing of the nuclear glasses with respect to alpha decay accumulation, involving directly alpha decays, but it is a heavy method to implement requiring experimental tools in hot cell facilities. Thus, such an approach is very expensive and the time needed to acquire the experimental data are quite long, typically several years. Moreover only few experimental techniques are available to characterize these radioactive samples. Consequently, only few studies have been performed with this method and the associated results are very important to validate the other methods of simulation of radiation ageing, which is one of the objectives of the current article. Moreover, up to now, only complex borosilicate glasses were studied by alpha decay self-irradiation, whose chemical complexity does not allow to implement some structural characterization techniques and thus to acquire structural data. ${ }^{1}$ Therefore, to improve the understanding of the nuclear oxide glasses evolution with respect to alpha decay self-irradiation, this study involving Cm-ISG started in 2008 at CEA-Marcoule DHAAtalante facility.

Knowledge about the ISG glass structural changes under alpha decay self-irradiation

This study has shown some evolutions of the Raman vibration bands with alpha decay dose, as described in the result section. In two previous studies performed on the same ISG-Cm glass, it was observed some evolutions of the local order of some cations, i.e.

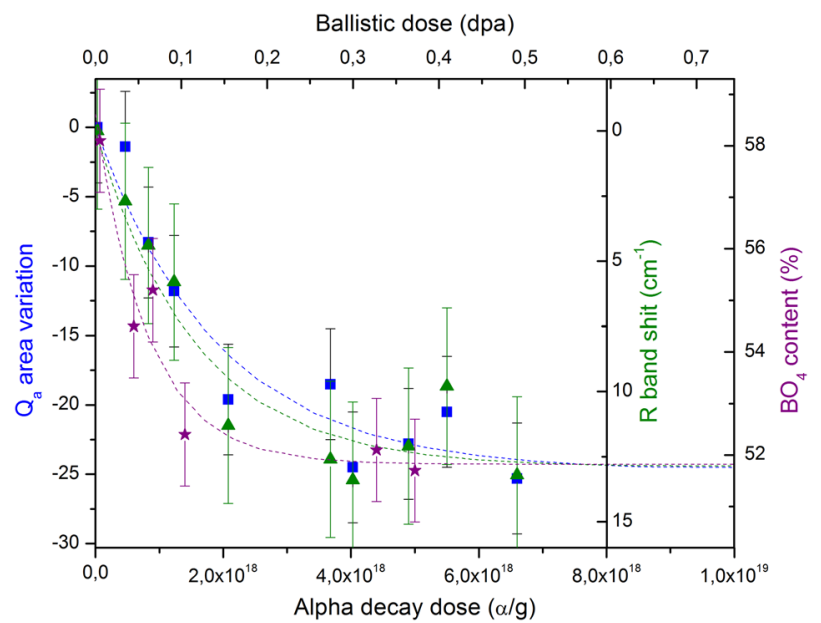

Fig. 5 Changes of structural parameters of Cm-ISG glass with alpha decay dose. 1 alpha decay per gram of glass corresponds to a nuclear dose of $1.6 \times 10^{-11} \mathrm{~Gy}$. The associated error bars represent the standard error associated to the data fits. The dotted lines are fits of experimental data with an exponential function. The impact parameters (previously called B) are respectively of $5.3 \times 10^{-19}, 6.4 \times$ $10^{-19}$, and $1.2 \times 10^{-18} \mathrm{~g}$ per alpha decay event for $Q_{a}$ area variation, $\mathrm{R}$ band shift and $\mathrm{BO}_{4}$ content variations, respectively

${ }^{11} \mathrm{~B},{ }^{29} \mathrm{Si},{ }^{27} \mathrm{Al}$, and ${ }^{23} \mathrm{Na}$ by NMR spectroscopy ${ }^{6}$ and $\mathrm{Zr}$, Pu, and $\mathrm{Cm}$ by Xanes spectroscopy. ${ }^{7}$ Therefore, both the short range and medium range orders of the glass are affected by alpha decay selfirradiation. The main short range order changes are a decrease by around $7 \%$ of the mean boron coordination number (higher boron content in trigonal configuration in the irradiated glass and lower boron content in tetrahedral configuration, Fig. 5), an increase of the local disorder around the $\mathrm{Al}, \mathrm{Zr}$ and $\mathrm{Na}$ sites, and a modification of the Si site. The nature of the latter change is not clearly identified because ${ }^{29} \mathrm{Si}$ NMR spectra did not evolve significantly (see supplementary information in ref. ${ }^{6}$ ); whereas, the $Q_{n}$ band changed in Raman spectra, mainly with a decrease of the $\mathrm{Q}_{a}$ contribution, that we assigned to $\mathrm{Q}_{4}(2 \mathrm{~T})$ units (Fig. 5). This change could be correlated to the evolution of $\mathrm{Zr}$ site with irradiation as observed by Bouty et al., ${ }^{7}$ with an increase of the seven fold coordinated site with respect to the octahedral site. A specific configuration of $\mathrm{SiO}_{4}$ tetrahedral around the $\mathrm{Zr}$ octahedral could thus be the origin of the $\mathrm{Q}_{a}$ band and its decrease with irradiation.

From molecular dynamic simulation studies of ballistic collisions in simplified borosilicate glasses, ${ }^{36,37}$ it was proposed that the main local order changes were associated to the partial conversion of boron from a tetrahedral to a trigonal configuration, that liberated some $\mathrm{Na}$ atoms (actually acting as charge compensators in the pristine glass), to act as modifier elements, and therefore increased the non-bridging oxygen (NBO) concentration in the glassy network. The experimental results discussed above only partially validate this description by showing the decrease of the mean boron coordination number; but, from the evolution of the ${ }^{29} \mathrm{Si}$ NMR and Raman spectra, it is not obvious to confirm such a description, the changes of NBO concentration estimated (due to boron coordination changes) at around $1 \%$ are certainly too small to be correctly detected.

Concerning the medium range order modifications with alpha decay self-irradiation, the main detected changes are a decrease of the mean $\mathrm{Si}-\mathrm{O}-\mathrm{Si}$ angle ( $\mathrm{R}$ band shift measured in the present study and described in Fig. 5 versus alpha decay dose) and an increase of the three membered silica rings (i.e. increase of the $D_{2}$ band in Raman spectra, as described in ref. ${ }^{4}$ ). These effects are 
qualitatively in good agreement with the results of molecular dynamic simulation of ballistic effects. ${ }^{37}$

Variation with alpha decay dose and damage model of Cm-ISG glass

The variation of the structural and macroscopic parameters of $\mathrm{Cm}$ ISG glass with alpha decay dose always follows an exponential law (Fig. 5 for structural parameter variations) which is agreement with all the results already obtained on actinide doped complex nuclear glasses. 19,20 This model of glass transformation corresponds to a direct impact damage meaning that only one passage of an alpha decay event is sufficient to damage at saturation the volume touched by the projectile, a further passage at the same location of an alpha decay event does not damage anymore the glass. The impact parameter (B) of the exponential law informs about the damaged volume of the alpha decay event (with $B=$ $\rho \times V$ and $\rho$ expressed in $\mathrm{g} \mathrm{nm}^{-3}$ ). From the fits of the experimental data variation with alpha decay dose, damage volumes of respectively $210 \pm 52,253 \pm 63,473 \pm 158,247 \pm 80$, and $538 \pm 92 \mathrm{~nm}^{3}$ were obtained for the $Q_{a}$ decrease, $R$ band shift, $\mathrm{BO}_{4}$ content variation, density and hardness changes, respectively. According to the uncertainty, these values seem quite similar with a mean value of around $345 \mathrm{~nm}^{3}$. This volume is very close to the damaged volume of a displacements cascade induced by a heavy projectile of $97 \mathrm{keV}$ (recoil nuclei energy of the ${ }^{244} \mathrm{Cm}$ alpha decay), determined by molecular dynamic simulation in a simple sodium borosilicate glass, ${ }^{31}$ i.e. $270 \mathrm{~nm}^{3}$. It suggests that the variation with alpha decay dose of the macroscopic properties and structural parameters of Cm-ISG are controlled by the accumulation of the damage induced by the recoil nuclei of the alpha decays.

As presented in the result section, this is also supported by the same variation with nuclear dose of properties and structural parameters of both Cm-ISG and Au-ISG glasses, leading to the conclusion that the variation with alpha decay dose of Cm-ISG glass is the result of the accumulation of the damage induced by the recoil nuclei of the alpha decay events. It supports the model of ballistic fast quenching events previously proposed because of the increase of glass fictive temperature with alpha decay selfirradiation of ${ }^{244} \mathrm{Cm}$-doped SON68 glass. ${ }^{38}$ In this view, the glass response to a recoil nuclei event is the result of the very fast quenching of the damaged zone in the displacement cascade. Therefore an analogy between the effects of the ballistic damage and the one of a very high-quenching rate from a melt equilibrated at very high temperature has been proposed and discussed. $^{36,39}$ To go further in this aspect, it is interesting to compare the Raman spectra of the irradiated ISG glasses with the high temperature $\left(700^{\circ} \mathrm{C}\right)$ ISG Raman spectra recently published. ${ }^{40}$ A very similar change of the $Q_{n}$ band and the appearance of $D_{2}$ band are observed in both studies. On the contrary, the $\mathrm{R}$ band shift is opposite, with a decrease of around $7 \mathrm{~cm}^{-1}$ with the increase of temperature up to $700^{\circ} \mathrm{C}$, and an increase of the $\mathrm{R}$ band with irradiation as described above (Fig. 5). Therefore, irradiation effects do not seem to be a pure thermal effect, which is not surprising due to the very short timescale of the displacement cascade (several ps) that certainly does not allow the damaged glass to reach an equilibrium state.

Simulation of alpha decay self-irradiation by heavy ions irradiation A second objective of this study is to evaluate how accurate is the use of heavy ions irradiations to simulate the consequences of alpha decay accumulation. As presented in the result section, the evolution with nuclear dose for both types of irradiations are very similar (same B parameters), showing the interest to use heavy ions in the MeV energy range. Nevertheless, the damage level of the $\mathrm{Au}$ ion irradiated glass is higher than the one of Cm-ISG glass (A parameters). This was also observed by Charpentier ${ }^{6}$ by comparing NMR spectra of Cm-ISG glass and heavy ions irradiated ISG glasses. These differences are certainly explained by the role of alpha particles involved in the alpha decay events as recently suggested by Mir on silica and sodium borosilicate glasses, ${ }^{41-43}$ and Karakurt and Charpentier on ISG glass., ${ }^{6,8}$ In particular, by using sequential irradiation with $\mathrm{Au}$ and $\mathrm{He}$ ions, Mir demonstrated that the alpha particles induced a partial healing of the damage previously generated by Au ions. Therefore, in an alpha decay event, associating both recoil nuclei and alpha particles, the final damaged state of the material is certainly the combination of the damage generated by the recoil nuclei and the partial healing induced by the alpha particles. ${ }^{6}$ In conclusion, the simulation of alpha decay self-irradiation by heavy ions irradiation is not fully representative in term of the amplitude of the glass transformation but offers an easier way to simulate an overestimate damaged state of the alpha doped case.

Implication for long-term behavior prediction of nuclear glasses This study has shown the relevance of using ISG glass to simulate the alpha decay self-irradiation ageing of complex SON68 glass, because of similar macroscopic variations observed on ${ }^{244} \mathrm{Cm}-$ doped glasses with alpha decay dose. Moreover, the increase of the altered layer thickness of the Au ions irradiated glass shows for the first time the importance of the structural state of the glass on its long-term leaching behavior. As recently reviewed by Gin et al., ${ }^{1}$ the level of alpha decay sustained by a nuclear glass before water arrival (expected at around several thousand years) will overpass the dose needed to damage significantly the glass (several $10^{18} \mathrm{a} / \mathrm{g}$ ). Therefore, the leaching studies, as undertaken currently, have to take into account the glass damage to predict as accurately as possible its long-term behavior. Further studies on all the leaching steps would improve the understanding of the glass response to radiation damage, and could assess if all the leaching mechanisms are impacted at the same level, as until now no impact of the alpha decay dose was quantified on SON68 glass from the initial alteration rate values. ${ }^{34,35}$ Furthermore, the longterm Cm-ISG glass chemical durability must be assessed and compared to the present results obtained from externally irradiated samples in order to complete the knowledge on the representativeness of alpha decay self-irradiation by heavy ion irradiation.

\section{Concluding remarks.}

- The ISG glass is a good surrogate of the SON68 glass under alpha self-irradiation in terms of both macroscopic property changes and structural evolutions. Moreover, these changes observed with alpha decay self-irradiation accumulation are primarily due to the ballistic effects of the recoil nuclei, which are offset by some partial annealing effects by the alpha particle.

- Structural evolution of the ISG glass has been assessed (Raman, NMR, and Xanes spectroscopies) and thus, it reinforces the mechanistic concepts concerning the glass response under alpha self-irradiation damage, i.e. a ballistic fast quenching event in the track of the recoil nucleus.

- Heavy ion irradiation in the MeV energy range allows to correctly represent the glass evolution with nuclear dose but this simulation of alpha decay self-irradiation is not fully representative in term of the amplitude of the glass transformation: it overestimates the damaged state as it does not take into account the partial healing induced by the alpha particles.

- The ISG glass altered thickness at high-reaction progress is impacted by the glass damage, as its structural and macroscopic properties. It can thus be concluded than the long-term ISG glass chemical durability depends on the glass 
Table 1. Theoretical glass compositions (\%wt oxides) of the two studied glasses

\begin{tabular}{lllllllll}
\hline \%wt oxides & $\mathrm{SiO}_{2}$ & $\mathrm{~B}_{2} \mathrm{O}_{3}$ & $\mathrm{Na}_{2} \mathrm{O}$ & $\mathrm{CaO}$ & $\mathrm{ZrO}_{2}$ & $\mathrm{Al}_{2} \mathrm{O}_{3}$ & $\mathrm{Cm}_{2} \mathrm{O}_{3}$ & $\mathrm{PuO}_{2}$ \\
\hline Non-radioactive ISG & 56.18 & 17.33 & 12.17 & 4.98 & 3.28 & 6.06 & 6.03 & 1.02 \\
$244 \mathrm{Cm}$ - doped ISG & 55.32 & 17.07 & 12.00 & 5.01 & 3.26 & 0.29 \\
\hline In the ${ }^{244} \mathrm{Cm}$-doped glass, the plutonium was formed from the alpha decay of ${ }^{244} \mathrm{Cm}$ in the initial $\mathrm{Cm}$ powder used to prepare the glass \\
\hline
\end{tabular}

structure. The level of alpha decay sustained by a nuclear glass before water arrival will overpass the dose needed to damage significantly the glass, i.e. several $10^{18} \mathrm{a} / \mathrm{g}$. For this reason the leaching studies, as undertaken currently, have to take into account the glass damage to predict as accurately as possible its long-term behavior.

\section{METHODS}

Glass sample preparation

Two different batches of ISG glass were studied: one is non-radioactive, similar to the international batch but fabricated in CEA-Marcoule; the second one is a ${ }^{244} \mathrm{Cm}$-doped ISG glass material fabricated in DHA-Atalante hot cells facility of CEA-Marcoule (Table 1).

The non-radioactive glass was synthetized by melting appropriate amounts of analytical grade of $\mathrm{SiO}_{2}, \mathrm{H}_{3} \mathrm{BO}_{3}, \mathrm{Na}_{2} \mathrm{CO}_{3}, \mathrm{CaO}, \mathrm{ZrO}_{2}$, and $\mathrm{Al}_{2} \mathrm{O}_{3}$. Mixed powders were melted in a Pt-Rh crucible for $3 \mathrm{~h}$ at $1300^{\circ} \mathrm{C}$. The fused glass was poured into graphite crucibles and annealed for $1 \mathrm{~h}$ at $600^{\circ} \mathrm{C}$, followed by slow cooling. Monoliths were cut from the glass blocks and polished to obtain a surface roughness of $<1 \mu \mathrm{m}$.

The radioactive $\mathrm{Cm}$-ISG batch is an ISG glass doped with $0.7 \mathrm{wt} \%{ }^{244} \mathrm{Cm}$ (Cm-ISG), synthesized by melting oxides, carbonates and nitrates. The mixture was melted for $3 \mathrm{~h}$ at $1400^{\circ} \mathrm{C}$ without stirring, then quenched to a temperature slightly above the glass transition point $\left(600^{\circ} \mathrm{C}\right)$, from which it was cooled more slowly to release the residual stresses from the glass. The cylindrical glass rod of $15 \mathrm{~mm}$ of diameter was cut into $1.5 \mathrm{~mm}$ thick disks, and then optically polished.

The theoretical compositions in weight percent of oxides are given in ref. ${ }^{44}$ for both non-radioactive and ${ }^{244} \mathrm{Cm}$-doped glass compositions. The density, $\rho$, measured by hydrostatic weight, is respectively of $2.50 \mathrm{~g} \mathrm{~cm}^{-3}$ for non-radioactive samples and $2.53 \mathrm{~g} \mathrm{~cm}^{-3}$ for the ${ }^{244} \mathrm{Cm}$-doped samples after fabrication.

\section{Irradiation conditions}

$\mathrm{Au}$ ions irradiations. Monoliths of the non-radioactive ISG glass were irradiated with gold ions at low energies on the $2 \mathrm{MV}$ Aramis accelerator of the SCALP platform at CSNSM (Orsay, France) to create damages in the initial glass structure. ${ }^{28,34}$ Multiple-energy irradiation was used to induce a constant deposited nuclear energy (nuclear damage) within the first micrometer of the irradiated samples with respective energies and fluence contribution (\% in bracket) of $0.5 \mathrm{MeV}(12 \%), 1.2 \mathrm{MeV}(12 \%), 2 \mathrm{MeV}(12 \%)$, and $3.5 \mathrm{MeV}(64 \%)$. The irradiation fluences range from $1.9 \times 10^{12}$ to $5.5 \times$ $10^{14}$ ions $/ \mathrm{cm}^{2}$. Gold implantation remains weak $(\leq 0.02 \mathrm{at} \%)$ in order to prevent from structural modifications by gold atoms. During irradiation, an aluminum foil recovered a part of the glass monoliths to keep a nonirradiated zone as a reference.

Alpha decay self-irradiation. The Cm-ISG glass was self-irradiated by the alpha decays of the ${ }^{244} \mathrm{Cm}$ atoms dispersed in its structure. Each alpha decay produces an alpha particle with a mean energy of around $5.8 \mathrm{MeV}$ and a recoil nucleus of ${ }^{240} \mathrm{Pu}$ of $97 \mathrm{keV}$. The alpha decay dose in Cm-ISG increases regularly with the storage time. Therefore, the samples were analyzed periodically to evaluate the properties change with alpha decay dose, which reaches around $6 \times 10^{18} \mathrm{a} / \mathrm{g}$ after around 8 years of glass storage.

So as to compare the material damage caused by the two irradiation ageing scenarios, the properties change have been compared by considering the deposited nuclear or electronic doses induced by both irradiation conditions.

\section{Leaching conditions}

Au-ISG glass monoliths were placed (irradiated side up) in the bottom of a PFA-Teflon reactor. Then a glass powder bed (grain sizes ranging 40-63 $\mu \mathrm{m}$ ) was placed over monoliths and pure water was added, in order to set the glass-surface-area-to-solution-volume ratio $(\mathrm{S} / \mathrm{V})$ at $200 \mathrm{~cm}^{-1}$ and thus to focus on the residual alteration rate regime. Leaching experiments were conducted in static mode, at $90^{\circ} \mathrm{C}$ for about 3 months; the $\mathrm{pH}$ was not buffered and quickly stabilized at about $(9.0 \pm$ $0.1)$. Glass monoliths irradiated at different fluences were regularly sampled to perform TOF-SIMS analysis.

\section{Solid characterizations}

Hardness measurements. The $\mathrm{Cm}$-ISG glass hardness was measured in air by Vickers micro-indentation in the DHA-Atalante hot cells facility. Details about the methodology are described in reference. ${ }^{31} 150 \mathrm{~g}$ load with a dwell time of $10 \mathrm{~s}$ was used.

The Au-ISG glass hardness was measured by nano-indentation with a Berkovich diamond indenter according to the methodology described in ref. ${ }^{28}$. The use of the continuous stiffness mode allowed the determination of the hardness variation versus the plastic penetration depth. The hardness of the irradiated layer is extracted at a penetration depth of $300 \mathrm{~nm}$. At higher depth the hardness measurement has not been considered because it reflects a mix of the hardness of the irradiated layer and of the underlying non-irradiated glass.

Density measurements. The density of the Cm-ISG glass was measured by means of a hydrostatic balance indentation in the DHA-Atalante hot cells facility. The glass sample was weighed in air, then in water according to Archimede's principle. The sample density represents the mean value of ten measurements.

On the Au-ISG irradiated glasses, the density evolution after gold ion irradiation was estimated from the step height measured by optical interferometry at the interface between the irradiated area and the nonirradiated one. The value considered is the mean value of 18 measurements for each fluence. We assume that the density change can be calculated considering the measured step height (in $\mathrm{nm}$ ) and a mean damage thickness of $1120 \mathrm{~nm}$.

ToF-SIMS. Elemental depth profiles were analyzed on monoliths by ToFSIMS, either to check the Au ions range in Au-ISG glasses (negative ions analysis, $\mathrm{Cs}^{+}$ion abrasion beam and $\mathrm{Bi}^{+}$ion primary beam) or to investigate glass or solution elements profiles in the Au-ISG alteration layer (positive ions analysis, $\mathrm{O}_{2}{ }^{+}$ion abrasion beam and $\mathrm{Bi}^{+}$ion primary beam). They were acquired on an IONTOF TOF 5 device (Tescan Analytics, Fuveau, France).

Raman spectroscopy. Horiba LabRAM HR800 micro-Raman spectrometer with a $532 \mathrm{~nm}$ excitation laser (green) and a $\times 100$ objective was used for non-polarized confocal Raman spectroscopy.

On non-radioactive samples (Au-ISG), the Raman spectra were obtained with a total acquisition time of $300 \mathrm{~s}$ ( $30 \mathrm{~s}$ per spectrum and 10 successive acquisitions to reduce spurious noise) and a 600 grooves per $\mathrm{mm}$ grating. Slit and confocal hole were settled to $100 \mu \mathrm{m}$ and $200 \mu \mathrm{m}$, respectively.

To record the spectra of Cm-ISG glass, the spectrometer was coupled with optical fibers to an optical microscope situated in a hot cell of DHAAtalante facility. The signal was also collected through a $\times 100$ objective. The data presented in Fig. 2a, b are raw Raman spectra without any background correction or treatment. To fit the $Q_{n}$ band, a linear baseline in between 850 and $1220 \mathrm{~cm}^{-1}$ was used. 
Data availability

The data that support the findings of this study are available from the corresponding author upon reasonable request.

\section{ACKNOWLEDGEMENTS}

The authors would like to thank the DHA technicians (Atalante facility) for fabricating and characterizing the ISG glasses, Cyril Bachelet (Nuclear and Mass Spectrometry Centre/CNRS, France) for carrying out the Au ion irradiations, and Laurent Dupuy (Tescan Analytics, Fuveau, France) for ToF-SIMS characterizations and Sophie Pavan and Jean-Luc Loubet (Ecole Cntrale de Lyon, Lyon, France) for nano-indentation measurements. This work was supported by Orano, EDF, and CEA.

\section{AUTHOR CONTRIBUTIONS}

S.P. and M.T. supervised the study. S.P. and S.Miro were responsible for experimental solid characterizations and M.T. and S.Mougnaud in charge of leaching experiments and associated characterizations. S.P., M.T., S.Mougnaud, and C.J. were involved in data interpretation. S.P. and M.T. wrote the paper. All the authors helped on paper editing.

\section{ADDITIONAL INFORMATION}

Competing interests: The authors declare no competing interests.

Publisher's note: Springer Nature remains neutral with regard to jurisdictional claims in published maps and institutional affiliations.

\section{REFERENCES}

1. Gin, S., Jollivet, P., Tribet, M., Peuget, S. \& Schuller, S. Radionuclides containmen in nuclear glasses: an overview. Radiochim. Acta https://doi.org/10.1515/ract-20162658 (2017).

2. Gin, S. et al. An international initiative on long-term behavior of high-level nuclear waste glass. Mater. Today 16, 243-248 (2013).

3. Mendoza, C., Peuget, S., Bouty, O., Caraballo, R. \& Jegou, C. Simplified nuclear glasses structure behaviour under various irradiation conditions: a Raman Spectroscopy Study. Procedia Chem. 7, 581-586 (2012).

4. Mendoza, $C$. et al. Oxide glass structure evolution under swift heavy ion irradiation. Nucl. Instrum. Methods Phys. Res. Sect. B: Beam Interact. Mater. At. 325, 54-65 (2014).

5. Peuget, S. et al. Alpha decays impact on nuclear glass structure. Procedia Mater. Sci. 7, 252-261 (2014).

6. Charpentier, T. et al. Self-healing capacity of nuclear glass observed by NMR spectroscopy. Sci. Rep. 6, https://doi.org/10.1038/srep25499 (2016).

7. Bouty, O., Ramond, L., Solari, P. L. \& Cammelli, S. XANES analysis of a Cm-doped borosilicate glass under alpha-self-irradiation effects. J. Mater. Sci. 51, 7918-7928 (2016).

8. Karakurt, G. et al. Understanding of the mechanical and structural changes induced by alpha particles and heavy ions in the French simulated nuclear waste glass. J. Nucl. Mater. 475, 243-254 (2016).

9. Inagaki, Y., Kikunaga, T., Idemitsu, K. \& Arima, T. Initial dissolution rate of the international simple glass as a function of $\mathrm{pH}$ and temperature measured using microchannel flow-through test method. Int. J. Appl. Glass Sci. 4, 317-327 (2013).

10. Gin, S. et al. Atom-probe tomography, TEM and ToF-SIMS study of borosilicate glass alteration rim: a multiscale approach to investigating rate-limiting mechanisms. Geochim. Et. Cosmochim. Acta 202, 57-76 (2017).

11. Gin, S. et al. Origin and consequences of silicate glass passivation by surface layers. Nat. Commun. 6, https://doi.org/10.1038/ncomms7360 (2015).

12. Gin, S. et al. The fate of silicon during glass corrosion under alkaline conditions: a mechanistic and kinetic study with the International Simple Glass. Geochim. Et. Cosmochim. Acta 151, 68-85 (2015).

13. Zhang, J. D. et al. Nanoscale imaging of alteration layers of corroded international simple glass particles using ToF-SIMS. Nucl. Instrum. Methods Phys. Res. Sect. BBeam Interact. Mater. At. 404, 45-51 (2017).

14. Reiser, J. T. et al. The use of positrons to survey alteration layers on synthetic nuclear waste glasses. J. Nucl. Mater. 490, 75-84 (2017).

15. Trivelpiece, C. L. et al. Corrosion of ISG fibers in alkaline solutions. J. Am. Ceram. Soc. 100, 4533-4547 (2017).

16. Thorat, V. S. et al. Corrosion of borosilicate glasses subjected to aggressive test conditions: structural investigations. J. Am. Ceram. Soc. 99, 3251-3259 (2016).
17. Fournier, M., Frugier, P. \& Gin, S. Resumption of alteration at high temperature and $\mathrm{pH}$ : rates measurements and comparison with initial rates. Procedia Mater. Sci. 7, 202-208 (2014).

18. Collin, M. et al. Structure of international simple glass and properties of passivating layer formed in circumneutral pH conditions. npj Mater. Degrad. 2, https:// doi.org/10.1038/s41529-017-0025-y (2018).

19. Weber, W. J. et al. Radiation effects in glasses used for immobilization of highlevel waste and plutonium disposition. J. Mater. Soc. 12, 1946-1978 (1997).

20. Peuget, S., Delaye, J. M. \& Jégou, C. Specific outcomes of the research on the radiation stability of the French nuclear glass towards alpha decay accumulation. J. Nucl. Mater. 444, 76-91 (2014).

21. Ziegler, J. F., Biersack, J. P. \& Ziegler, M. D. SRIM, the stopping and range of ions in matter. (Chester, Maryland, SRIM Co., 2008).

22. Moll, S. et al. Comparison between simulated and experimental Au-ion profiles implanted in nanocrystalline ceria. Nucl. Instrum. Methods Phys. Res. Sect. B: Beam Interact. Mater. At. 307, 93-97 (2013).

23. Furukawa, T. F., Karen, E. Fox., \& White, William B. Raman spectroscopic investigation of the structure of silicate glasses. III. Raman intensities and structural units in sodium silicate glasses.The Journal of Chemical Physics 75, 3226-3237 (1981).

24. Umari, P., Gonze, X. \& Pasquarello, A. Concentration of small ring structures in vitreous silica from a first-principles analysis of the Raman Spectrum. Phys. Rev. Lett. 90, 027401 (2003).

25. McMillan, P. Structural studies of silÿicate glasses and melts - applications and limitations of Raman spectroscopy. Am. Mineral. 69, 622-644 (1984).

26. Mysen, B. O., Finger, L. W., Virgo, D., \& Seifert, F. A. Curve-fitting of Raman spectra of silicate glasses. Am. Mineral. 67, 686-695 (1982).

27. Bouty, O., Cammelli, S. \& Solari, P. L. Structural insights for the International Simple Glass by combining X-ray absorption spectroscopic analysis and atomistic modelling. J. Non-Cryst. Solids. https://doi.org/10.1016/j.jnoncrysol.2017.11.013 (2017).

28. Peuget, S. et al. Effects of deposited nuclear and electronic energy on the hardness of R7T7-type containment glass. Nucl. Instrum. Methods Phys. Res. Sect. B-Beam Interact. Mater. At. 246, 379-386, https://doi.org/10.1016/j. nimb.2005.12.053 (2006).

29. Matzke, H. in Université d'été du CEA-Valrhô. (Méjannes Le Clap (France), Odyssée).

30. Inagaki, Y. et al. Microstructure of simulated high-level waste glass doped with shortlived actinides, 238Pu and 244Cm. Vol. 257 199-206 (Materials Research Society, Pittsburgh, PA, 1992).

31. Peuget, S. et al. Irradiation stability of R7T7-type borosilicate glass. J. Nucl. Mater 354, 1-13, https://doi.org/10.1016/j.nucmat.2006.01.021 (2006).

32. Inagaki, Y. et al. Effects of alpha-decay on mechanical properties of simulated nuclear waste glass.Sci. Basis Nuclear Waste Manag XVI Symp 294, 191-198 (1992).

33. Scheetz, B. E. et al. The role of boron in monitoring the leaching of borosilicate glass waste forms.Scientific Basis for Nuclear Waste Management VIII. Proceedings of the Eighth International Symposium 44, 129-134 (1985).

34. Peuget, S. et al. Effect of alpha radiation on the leaching behaviour of nuclear glass. Journal of Nuclear Materials 362, 474-479, https://doi.org/10.1016/j. jnucmat.2007.01.099 (2007).

35. Tribet, M. et al. Irradiation impact on the leaching behavior of HLW glasses. Procedia Mater. Sci. 7, 209-215 (2014).

36. Delaye, J. M., Peuget, S., Calas, G., \& Galoisy, L. Comparative effects of thermal quenching and ballistic collisions in $\mathrm{SiO}_{2}-\mathrm{B}_{2} \mathrm{O}_{3}-\mathrm{Na}_{2} \mathrm{O}$ glass. Nucl. Instrum. Methods Phys. Res. Sect. B-Beam Interact. Mater. At. 326, 256-259, https://doi.org/ 10.1016/j.nimb.2013.10.061 (2014).

37. Delaye, J. M., Peuget, S., Bureau, G. \& Calas, G. Molecular dynamics simulation of radiation damage in glasses. J. Non-Cryst. Solids 357, 2763-2768 (2011).

38. Maugeri, E. A. et al. Calorimetric study of glass structure modification induced by a decay. J. Am. Ceram. Soc. 95, 2869-2875 (2012).

39. Peuget, $S$. et al. Comparison of radiation and quenching rate effects on the structure of a sodium borosilicate glass. J. Non-Cryst. Solids 378, 201-212 (2013).

40. Guerette, M. \& Huang, L. P. In-situ Raman and Brillouin light scattering study of the international simple glass in response to temperature and pressure. J. NonCryst. Solids 411, 101-105 (2015).

41. Mir, A. H. et al. Understanding and simulating the material behavior during multiparticle irradiations. Sci. Rep. 6, 11 (2016).

42. Mir, A. H. et al. Defect recovery and damage reduction in borosilicate glasses under double ion beam irradiation. Epl 112, https://doi.org/10.1209/0295-5075/ 112/36002 (2015).

43. Mir, A. H. et al. Mono and sequential ion irradiation induced damage formation and damage recovery in oxide glasses: stopping power dependence of the mechanical properties. J. Nucl. Mater. 469, 244-250 (2016).

44. Mendoza, C., Peuget, S., Bouty, O., Caraballo, R. \& Jegou, C. in Atalante2012 International Conference on Nuclear Chemistry for Sustainable Fuel Cycles Vol. 7 Procedia Chemistry (C. Poinssot ed) 581-586 (Elsevier Science Bv, 2012). 
45. Le Gac, A. Comportement sous irradiation des verres d'aluminosilicates, Thesis of Paris-Saclay University, France, (2017).

46. Mir, A. H. Radiation effects on oxide glasses: importance of energy deposition and relaxation processes, Thesis of Caen University, France, (2015).

(c) Open Access This article is licensed under a Creative Commons Attribution 4.0 International License, which permits use, sharing, adaptation, distribution and reproduction in any medium or format, as long as you give appropriate credit to the original author(s) and the source, provide a link to the Creative
Commons license, and indicate if changes were made. The images or other third party material in this article are included in the article's Creative Commons license, unless indicated otherwise in a credit line to the material. If material is not included in the article's Creative Commons license and your intended use is not permitted by statutory regulation or exceeds the permitted use, you will need to obtain permission directly from the copyright holder. To view a copy of this license, visit http://creativecommons. org/licenses/by/4.0/.

c) The Author(s) 2018 